\title{
An updated meta-analysis of the prognostic value of decreased E-cadherin expression in ovarian cancer
}

\author{
LiLi Yu' ${ }^{1}$ Xiaoli Hua ${ }^{2}$, Yu Yang ${ }^{2}, \mathrm{Ke} \mathrm{Li}^{2}$, Qilin Zhang ${ }^{2}$ and Lixiu Yu ${ }^{2}$ \\ ${ }^{1}$ Department of Obstetrics and Gynecology, Union Hospital, Tongji Medical College, Huazhong University of Science and \\ Technology, Wuhan 430022, PR China \\ ${ }^{2}$ Department of Pharmacy, Union Hospital, Tongji Medical College, Huazhong University of Science and Technology, Wuhan \\ 430022, PR China
}

Correspondence to: Lixiu Yu, email: ylx120515@163.com

Keywords: ovarian cancer, E-cadherin, prognosis, updated meta-analysis

Received: March 22, 2017 Accepted: August 26, $2017 \quad$ Published: September 14, 2017

Copyright: Yu et al. This is an open-access article distributed under the terms of the Creative Commons Attribution License 3.0 (CC BY 3.0 ), which permits unrestricted use, distribution, and reproduction in any medium, provided the original author and source are credited.

\section{ABSTRACT}

Decreased epithelial cadherin (E-cadherin) expression is hypothesized to be related to poor prognosis of ovarian cancer, but the predictive value is still inconsistent. We conducted an updated meta-analysis with a total of 16 studies enrolling 1720 patients to estimate the prognostic value of decreased E-cadherin expression in ovarian cancer. Reduced expression of E-cadherin was significantly associated to poor overall survival ( $\mathrm{HR}=1.74,95 \% \mathrm{CI}: 1.40-2.17)$ and progression-free survival $(\mathrm{HR}=$ 1.45, 95\% CI: 1.12-1.86) with a large heterogeneity for overall survival. In addition, we found that decreased expression of E-cadherin was significantly correlated with International Federation of Gynecology and Obstetrics grade ( $\mathrm{HR}=3.74,95 \% \mathrm{CI}$ : 2.24-6.23), E-cadherin membranous ( $H R=1.47,95 \%$ CI: 1.01-2.14), pathologic grade $(H R=1.41,95 \%$ CI: 1.01-1.97), residual tumor size $(H R=2.72,95 \% \mathrm{CI}$ : 1.99-3.72), and surgery ( $H R=3.21,95 \% C I: 1.19-8.67)$. Our finding suggests that decreased E-cadherin expression may be a predictor of poor ovarian cancer prognosis.

\section{INTRODUCTION}

Ovarian cancer threatens women's health worldwide as a lethal disease that is challenging to diagnose in early stages $[1,2]$. Although progress has been made in diagnostics and treatments of ovarian cancer, the prognosis of ovarian cancer patients is far from optimistic [2, 3]. The International Federation of Gynecology and Obstetrics (FIGO) stage, histotype and grade of differentiation are recognized as classical prognostic factors, but they cannot accurately predict the prognosis of ovarian cancer [4, 5]. However, studies have proved that many biomarkers are involved in the progression of ovarian cancer [6], identification and validation of prognostic factors can complement well-established clinical histopathology analysis with the aim of improving future treatments [7].

E-cadherin is a calcium dependent transmembrane glycoprotein, located in chromosome 16q22.1. Inhibition of E-cadherin function can lead to reduced cell proliferation and viability [8]. Decreased expression of E-cadherin can destroy the intracellular junction so that epithelial cells acquire the ability to migrate [9]. Therefore, impaired function of E-cadherin could lead to invasive potential and migration of malignant epithelial tumors. Previous studies have suggested that decreased expression of E-cadherin is closely related to the occurrence, differentiation, invasion, metastasis, and prognosis of tumors in ovarian, breast, gastric, and prostate cancer [9-13]. The possible mechanisms may be E-cadherin gene mutation, E-cadherin promoter hypermethylation, suppression of RNA transcription, or matriptase activation $[14,15]$. Studies also suggested that decreased E-cadherin expression was associated with high histological grade and deep myometrial invasion [16, 17]. However, some other studies drew different conclusions [18-20], so associations between decreased expression of E-cadherin and ovarian cancer prognosis are still debated. Previous reviews, including meta-analyses, did not explore associations between decreased expression of E-cadherin and progression-free survival (PFS) and histological grade 
in depth $[21,22]$. Considerable progress has been made in this area [23-26], so we conducted an updated metaanalysis to evaluate the relationship between E-cadherin and ovarian cancer prognosis comprehensively.

\section{RESULTS}

\section{Search results}

As shown in Figure 1, a total of 1053 citations were identified. Of these, 548 articles were removed for duplicated data. Through reviewing titles and abstracts, 422 articles were excluded due to irrelevant publications on E-cadherin expression in ovarian cancer. After systematically reading the remaining studies, 67 articles were excluded because the studies were in vitro or nonhuman studies, reviews, or did not report overall survival (OS) or progression-free survival (PFS) in ovarian cancer. A total of 16 articles with 1720 ovarian cancer patients [18-20, 23-35] were determined eligible.

\section{Study characteristics}

The main characteristics of the eligible studies are documented in Table 1. Of the 16 articles published from 1997 to 2017, thirteen articles were high-quality studies (NOS scores $\geq 7.0$ ). Seven studies presented the OS and PFS, while nine studies present OS only. The level of E-cadherin expression in ovarian cancer tissues was frequently detected by immunohistochemistry (IHC). The hazard ratio (HR) value was obtained directly or from survival curves of the studies.

\section{Influence of reduced E-cadherin expression on OS and PFS}

Pooled results of the 16 studies suggested that reduced expression of E-cadherin was significantly associated with OS $(\mathrm{HR}=1.74,95 \% \mathrm{CI}: 1.40-2.17)$ (Figure 2). Thus, ovarian cancer with reduced E-cadherin expression had a higher risk of mortality, but significant heterogeneity $\left(I^{2}=57.0 \%, p=0.003\right)$ was observed across the studies, then random effect model was used. We performed further subgroup analysis and metaregression to explain significant heterogeneity from six distinct sources, which are listed in Table 2. HR extracted directly from the articles was 2.08 (95\% CI: 1.47-2.95) with significant heterogeneity $\left(I^{2}=52.0 \%, p=0.022\right)$ (Figure 2; Directly obtained). The pooled HR obtained from the Kaplan-Meier curves was 1.37 (95\% CI: 1.16$1.63)$ with no significant heterogeneity $\left(I^{2}=21.2 \%, p=\right.$ 0.28 ) (Figure 2; From curves). We calculated pooled HRs for OS and PFS from multivariate or univariate criteria separately. In the multivariate HR estimation group, the pooled HR for OS was 2.30 (95\% CI: 1.62-3.26, $I^{2}=$ $71.6 \%, p<0.01)$, and the pooled HR for PFS was 2.52 (95\% CI: $1.33-4.78, I^{2}=0 \%, p=0.33$ ). Similarly, in the univariate HR estimation group the pooled HR for OS was

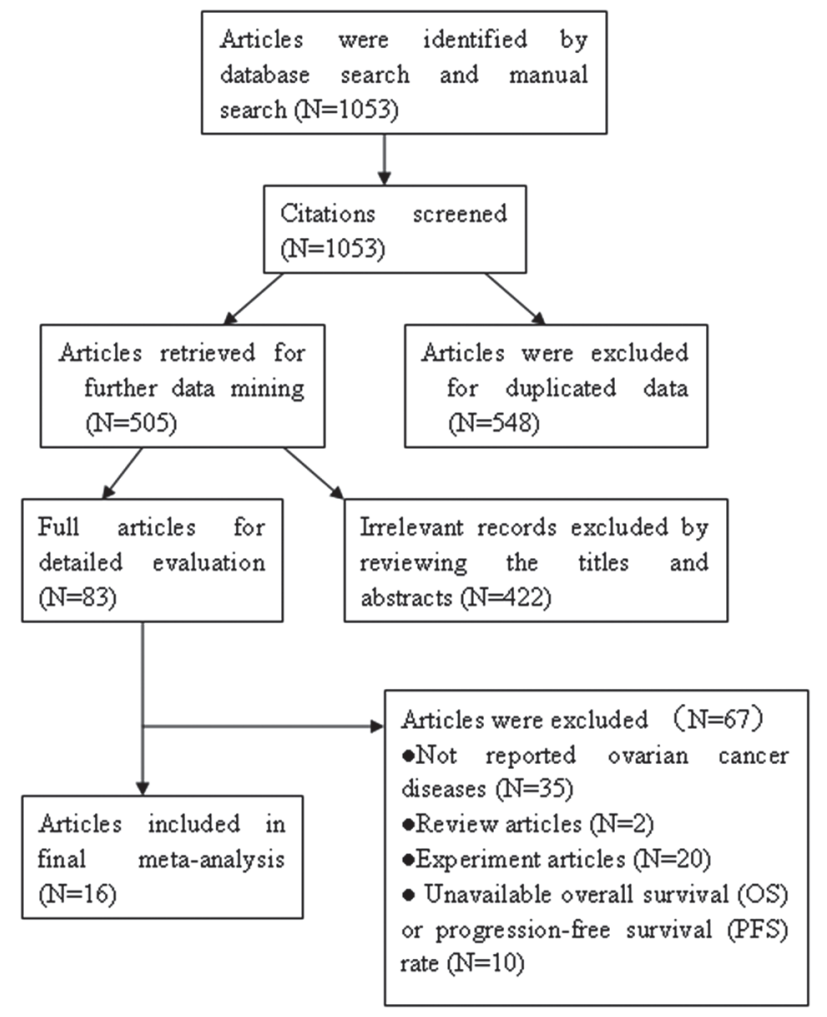

Figure 1: The flow diagram of retrieval in this study. 
Table 1: Study characteristics

\begin{tabular}{|c|c|c|c|c|c|c|c|c|c|c|c|}
\hline First author & Country & $\begin{array}{c}\text { Published } \\
\text { year }\end{array}$ & $\begin{array}{c}\text { Mean age } \\
\text { (year) }\end{array}$ & $\begin{array}{l}\text { FIGO } \\
\text { stage }\end{array}$ & Cases & $\begin{array}{l}\text { Mean Follow- } \\
\text { up (year) }\end{array}$ & $\begin{array}{c}\text { Cut-off } \\
\text { level (\%) }\end{array}$ & Method & $\begin{array}{c}\text { Survival } \\
\text { type }\end{array}$ & HR estimation & NOS \\
\hline Sundov D. [23] & Croatia & 2017 & 55 & I-VI & 81 & NA & 10 & $\mathrm{IHC}$ & OS & Directly obtained & 7 \\
\hline Juan W. [24] & China & 2016 & 53 & I-VI & 257 & 3 & NA & $\mathrm{IHC}$ & $\mathrm{OS} / \mathrm{PFS}$ & Directly obtained & 7 \\
\hline Li X. [25] & China & 2016 & 47 & I-VI & 43 & 2.12 & 20 & $\mathrm{IHC}$ & OS/PFS & Directly obtained & 7 \\
\hline Liew PL. [26] & Taiwan & 2015 & 54.3 & I-VI & 108 & 4.13 & 10 & $\mathrm{IHC}$ & OS/DFS & Directly obtained & 7 \\
\hline Bodnar L. [27] & Poland & 2014 & 54 & I-VI & 61 & NA & 10 & $\mathrm{IHC}$ & OS/ PFS & Directly obtained & 8 \\
\hline Taskin S. [28] & Ankara & 2012 & 58.63 & I-VI & 30 & 2.81 & 25 & $\mathrm{IHC}$ & OS & Directly obtained & 6 \\
\hline Huang KJ. [18] & China & 2012 & NA & NA & 136 & 2.13 & 5 & $\mathrm{IHC}$ & OS & Directly obtained & 7 \\
\hline Dian D. [19] & Germany & 2011 & 60.35 & I-VI & 100 & 13 & 25 & $\mathrm{IHC}$ & OS/ PFS & From curves & 7 \\
\hline Но CM. [29] & China & 2010 & 51 & II-VI & 58 & 2.72 & 10 & $\mathrm{IHC}$ & OS/PFS & Directly obtained & 6 \\
\hline Shim HS. [30] & Kroea & 2009 & 50.2 & II-VI & 72 & NA & 25 & $\mathrm{IHC}$ & OS & From curves & 7 \\
\hline Blechschmidt K. [31] & Germany & 2008 & 63 & III,IV & 48 & 4.58 & 10 & $\mathrm{IHC}$ & OS & Directly obtained & 8 \\
\hline Scholten AN. [32] & Netherlands & 2006 & NA & I-VI & 225 & 11.6 & 25 & $\mathrm{IHC}$ & OS & Directly obtained & 7 \\
\hline Cho EY. [33] & Korea & 2006 & NA & I-VI & 95 & 2.67 & 10 & $\mathrm{IHC}$ & OS & From curves & 6 \\
\hline Voutilainen KA. [20] & Finland & 2006 & 62 & I-VI & 282 & 12 & 5 & $\mathrm{IHC}$ & $\mathrm{OS} / \mathrm{PFS}$ & From curves & 8 \\
\hline Faleiro-Rodrigues C. [34] & Portugal & 2004 & 56 & I-VI & 104 & 6 & 10 & $\mathrm{IHC}$ & OS & Directly obtained & 8 \\
\hline Darai E. [35] & France & 1997 & 53.5 & I-III & 20 & 24 & 10 & $\mathrm{IHC}$ & OS & From curves & 7 \\
\hline
\end{tabular}

Note: NA: Not available; HR: Hazard ratio; FIGO: International Federation of Gynecology and Obestetrics; IHC: Immunohistochemistry; OS: Overall survival; PFS: Progressionfree survival; NOS:Newcastle-Ottawa quality assessment scale.

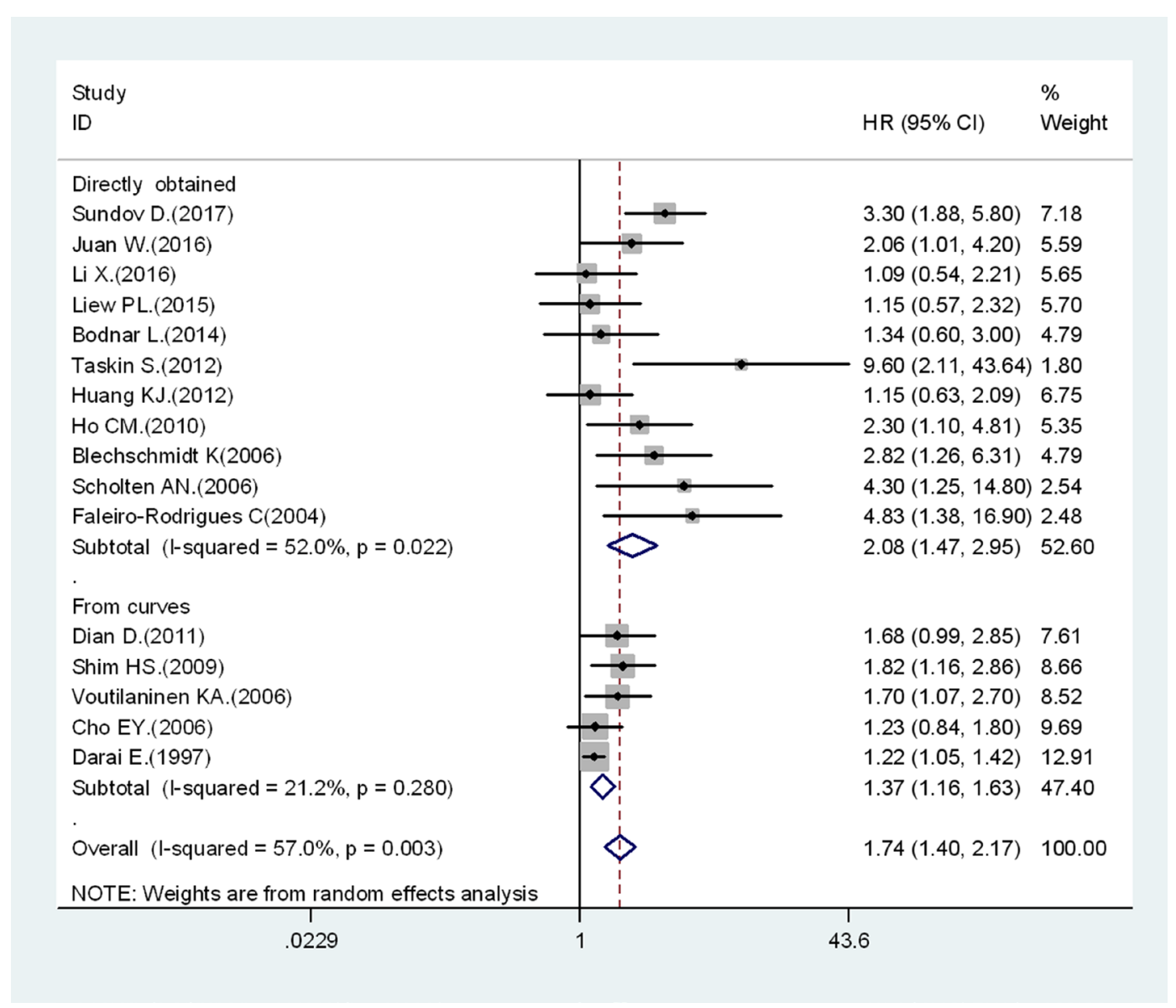

Figure 2: Association between decreased E-cadherin expression and OS stratified by HR estimation: directly obtained and from curves. 
1.38 (95\% CI: $\left.1.11-1.70, P^{2}=1.4 \%, p=0.42\right)$, and the pooled HR for PFS was 1.29 (95\% CI: $0.99-1.69, I^{2}=0 \%$, $p=0.50)$. The results indicated that there were significant associations between reduced expression of E-cadherin and OS and PFS, except for the univariate HR estimation group for PFS. The results further confirmed that patients with decreased expression of E-cadherin had poor OS in ovarian cancer. Considering the evaluation criteria of IHC, the pooled HR was 1.72 (95\% CI: $1.32-2.24)$ in the percentage group, 2.50 (95\% CI: $1.39-4.51)$ in the semiquantitative group, and 1.25 (95\% CI: $1.08-1.43)$ in the combined group. These results also suggested that the correlation between reduced expression of E-cadherin and poor OS was significant. Meta-regression analysis showed that there was no statistically significant difference among subgroups ( $p=0.364)$. In other stratified analyses by year, nation, article quality, and histological type, we found no statistically significant difference among subgroups as presented in Table 2.

The PFS analysis was based on 7 studies. The pooled results indicated that decreased expression of E-cadherin could prefigure poor PFS in ovarian cancer $(\mathrm{HR}=1.45,95 \% \mathrm{CI}: 1.12-1.86$, Figure 3$)$. There was no significant heterogeneity $\left(I^{2}=20.6 \%, p=0.273\right)$.

\section{Association between reduced E-cadherin expression and clinicopathological characteristics}

The pooled results for reduced E-cadherin expression and clinicopathological characteristics are illustrated in Table 3 . The results indicated that decreased expression of E-cadherin was significantly associated with FIGO stage (III-IV vs. I-II: HR = 3.74, 95\% CI: 2.24 6.23), surgery (suboptimal vs. optimal, $\mathrm{HR}=3.21,95 \%$ CI: $1.19-8.67)$, residual tumor $(\geq 1 \mathrm{~cm}$ vs. $<1 \mathrm{~cm}, \mathrm{HR}=$ 2.72, 95\% CI: 1.99-3.72), and E-cadherin membranous (negative vs. positive, $\mathrm{HR}=1.47,95 \% \mathrm{CI}$ : $1.01-2.14$ ) . However, the association between decreased E-cadherin expression and lymphatic metastasis (negative vs. positive: $\mathrm{HR}=1.40,95 \% \mathrm{CI}: 0.63-3.10$ ) or chemotherapy (nonpaclitaxel vs. paclitaxel: $\mathrm{HR}=1.31,95 \% \mathrm{CI}$ : $0.31-5.57$ ) was not significant.

\section{Sensitivity analysis}

Sensitivity analysis was conducted by sequential omission of each article to evaluate stability concerning OS and PFS. Exclusion of any single study did not alter the pooled HR estimation, which indicated that our results were robust (Figure 4).

\section{Publication bias}

Both Funnel plot and Egger's tests were used to evaluate the publication bias of included articles. Our results suggested that there was potential asymmetry in the studies on the association between reduced expression of E-cadherin and OS ( $p$ Egger $=0.002$ ), so a trim-andfill analysis was conducted. As shown in Figure 5A, after incorporation of six additional studies, the funnel plots were symmetrical, and E-cadherin low-expression was related to poor $\mathrm{OS}$ (corrected $\mathrm{HR}=1.37,95 \% \mathrm{CI}$ : 1.08-1.74). Figure 5B shows that all articles lay inside the $95 \%$ CIs, with an even distribution around the vertical. Egger's tests also suggested no significant publication bias $(p$ Egger $=0.70)$.

\section{DISCUSSION}

We conducted a comprehensive meta-analysis toward investigating the prognostic value of decreased E-cadherin expression in ovarian cancer. A total of 16 articles [18-20, 23-35] with 1720 enrolled patients were included in this meta-analysis. The results indicated that decreased expression of E-cadherin was significantly associated with poor OS (HR $=1.74,95 \% \mathrm{CI}: 1.40-2.17$, $\left.I^{2}=57.0 \%, p=0.003\right)$ and poor PFS $(\mathrm{HR}=1.45,95 \%$ CI: $1.12-1.86, I^{2}=20.6 \%, p=0.273$ ), suggesting that patients with decreased expression of E-cadherin have poor prognosis in ovarian cancer. Consistent with the conclusion that low expression of E-cadherin carries a worse prognosis [26, 29], E-cadherin may be an important target for the treatment of ovarian cancer.

We also assessed the associations between decreased E-cadherin expression and clinicopathological features. The results indicated that decreased expression of E-cadherin was significantly associated with high FIGO stage (III and IV) compared with low FIGO stage (I and II) $\left(\mathrm{HR}=3.74,95 \% \mathrm{CI}: 2.24-6.23, I^{2}=0, p=0.37\right)$. Reduced E-cadherin expression has significant association with residual tumor $(\geq 1 \mathrm{~cm}$ vs. $<1 \mathrm{~cm}, \mathrm{HR}=2.72,95 \%$ CI: 1.99-3.72), E-cadherin membranous staining (negative vs. positive, $\mathrm{HR}=1.47,95 \% \mathrm{CI}: 1.01-2.14$ ), and surgery (suboptimal vs. optimal, $\mathrm{HR}=3.21,95 \% \mathrm{CI}$ : 1.19-8.67). A significant association between decreased expression of E-cadherin and lymphatic metastasis (negative vs. positive: $\mathrm{HR}=1.40,95 \% \mathrm{CI}: 0.63-3.10)$ or chemotherapy (non-paclitaxel vs. paclitaxel: $\mathrm{HR}=1.31,95 \% \mathrm{CI}$ : $0.31-$ 5.57) was not found. Given the limited number of original studies of clinicopathological features, further studies are needed to confirm the association between decreased E-cadherin expression and clinicopathological.

To date, there is no reliable clinical predictor for ovarian cancer, but the decreased expression of E-cadherin has been reported as an important event in ovarian cancer invasion and metastasis. E-cadherin belongs to the calcium-dependent adhesion molecule protein family and is mainly distributed in the epithelial tissue [36]. The intact structure of normal ovarian surface epithelium is dependent on N-cadherin, while E-cadherin is hardly expressed. Compared with well-differentiated epithelial carcinoma the expression of E-cadherin is 
Table 2: The pooled data on overall survival of meta-analysis

\begin{tabular}{|c|c|c|c|c|c|}
\hline \multirow{2}{*}{ Subgroup analysis } & \multicolumn{2}{|c|}{ Pooled HR (95\% CI) } & \multirow{2}{*}{$\begin{array}{c}\text { Meta-regression } \\
(p \text { value })\end{array}$} & \multicolumn{2}{|c|}{ Heterogeneity } \\
\hline & Random-effect model & Fixed-effect model & & I2 value (\%) & $p$ value \\
\hline Year & & & 0.114 & & \\
\hline$\leq 2010$ & $1.69(1.30-2.20)$ & $1.37(1.21-1.55)$ & & 59.40 & 0.016 \\
\hline$>2010$ & $1.77(1.25-2.51)$ & $1.74(1.38-2.19)$ & & 52.50 & 0.032 \\
\hline Country & & & 0.094 & & \\
\hline Asia & $1.55(1.24-1.94)$ & $1.46(1.25-1.71)$ & & 33.80 & 0.15 \\
\hline Non-Asia & $2.26(1.42-3.61)$ & $1.43(1.23-1.66)$ & & 72.0 & 0.001 \\
\hline HR estimation & & & 0.036 & & \\
\hline From curves & $1.37(1.16-1.63)$ & $1.33(1.17,1.50)$ & & 52.0 & 0.02 \\
\hline Directly obtained & $2.08(1.47-2.95)$ & $1.92(1.53-2.40)$ & & 21.10 & 0.28 \\
\hline Scoring criteria & & & 0.364 & & \\
\hline Percentage & $1.72(1.32-2.24)$ & $1.69(1.37-2.09)$ & & 29.9 & 0.179 \\
\hline Semiquantitative & $2.50(1.39-4.51)$ & $2.46(1.70-3.55)$ & & 56.2 & 0.077 \\
\hline Combined & $1.25(1.08-1.43)$ & $1.25(1.08-1.43)$ & & 0.2 & 0.367 \\
\hline NOS & & & 0.819 & & \\
\hline 6 & $2.33(0.95-5.73)$ & $1.54(1.11-2.14)$ & & 75.3 & 0.017 \\
\hline 7 & $1.62(1.23-2.12)$ & $1.37(1.21-1.55)$ & & 58.3 & 0.014 \\
\hline 8 & $2.01(1.31-3.09)$ & $1.93(1.37-2.72)$ & & 24.9 & 0.26 \\
\hline Histological type & & & 0.425 & & \\
\hline Serious & $1.84(1.33-2.53)$ & $1.81(1.37-2.40)$ & & 18.4 & 0.29 \\
\hline
\end{tabular}

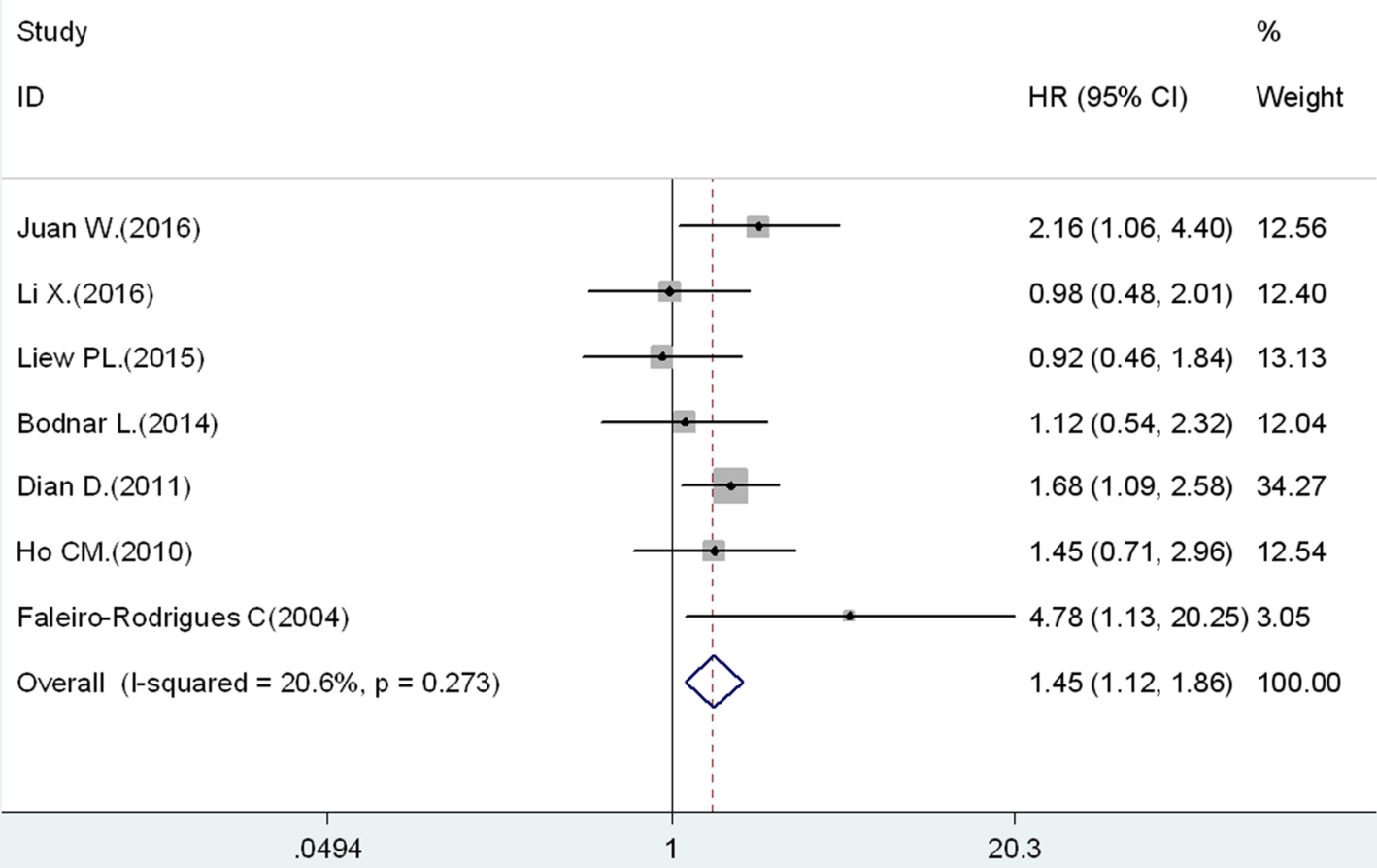

Figure 3: Results of association between decreased expression of E-cadherin and PFS. 
Table 3: Associations between decreased expression of E-cadherin and clinicopathological features

\begin{tabular}{|c|c|c|c|c|c|c|}
\hline \multirow{2}{*}{ Clinicopathological features } & \multirow{2}{*}{ Pooled HR } & \multicolumn{2}{|c|}{ 95\% CI for HR } & \multirow{2}{*}{$p$ value } & \multirow{2}{*}{$I^{2}$ value $(\%)$} & \multirow{2}{*}{ Model } \\
\hline & & Lower & Upper & & & \\
\hline Age $(\geq 50$ vs. $<50)$ & 1.07 & 0.85 & 1.36 & 0.145 & 41.5 & Random \\
\hline FIGO stage (III, IV vs. I, II ) & 3.74 & 2.24 & 6.23 & 0.37 & 0 & Fixed \\
\hline $\begin{array}{l}\text { E-cadherin membranous staining (negative vs. } \\
\text { positive) }\end{array}$ & 1.47 & 1.01 & 2.14 & 0.78 & 0 & Fixed \\
\hline Pathologic grade (G3 vs. G1, G2) & 1.41 & 1.01 & 1.97 & 0.13 & 47 & Random \\
\hline Residual tumor $(<1 \mathrm{~cm}$ vs. $>1 \mathrm{~cm})$ & 2.72 & 1.99 & 3.72 & 0.51 & 0 & Fixed \\
\hline Surgery ( suboptimal vs. optimal ) & 3.21 & 1.19 & 8.67 & 0.074 & 68.7 & Random \\
\hline Chemotherapy (non-paclitaxel vs. paclitaxel) & 1.31 & 0.31 & 5.57 & $<0.01$ & 90.4 & Random \\
\hline Lymphatic metastasis (negative vs. positive) & 1.40 & 0.63 & 3.10 & 0.01 & 86.6 & Random \\
\hline
\end{tabular}

usually decreased or absent in poorly-differentiated ovarian carcinoma. Previous studies demonstrated that loss of E-cadherin was the most important hallmark of epithelial-mesenchymal transition, which is implicated in the dissemination, migration, and invasion of cancer cells $[37,38]$. The loss of E-cadherin expression also has an inseparable association with chemotherapy resistance in cancer cells [39] and can cause tumor cells to present apparent properties of cancer stem cells [40]. However, Darai et al. demonstrated that decreased expression of E-cadherin was uncorrelated with cancer type, pathological grade and tumor size [35]. Point mutation and partial deletion of E-cadherin gene can also cause loss of cell adhesion while tyrosine phosphorylation in the E-cadherin-beta catenin complex can inhibit the function of E-cadherin without changing its expression. Further mechanistic research is warranted, these studies support our hypothesis that decreased of expression E-cadherin is a promising prognosis factor of survival in ovarian cancer.

Significant heterogeneity among studies $\left(I^{2}=57.0 \%\right.$, $p=0.003$ ) was found when survival data were pooled for OS. Subgroup analysis and meta-regression analysis were performed to explore the source of heterogeneity. The results suggested that methods for HR estimation might contain variables associated with this heterogeneity. Stratified analysis showed that the heterogeneity was less than $50 \%\left(I^{2}=21.2 \%, p=0.28\right)$ in HR obtained from curves group, yet there was significant heterogeneity $\left(I^{2}=52.0 \%, p=0.022\right)$ in HR directly extracted group. Similarly, stratified analysis by multivariate/ univariate criteria for HR estimation suggested that the heterogeneity was significant $\left(I^{2}=71.6 \%, p<0.01\right)$ in multivariate HR group. While, there was not significant heterogeneity $\left(I^{2}\right.$ $=1.4 \%, p=0.42$ ) in univariate HR group. Importantly, meta-regression analysis showed that there was significant heterogeneity among the HR estimation subgroup ( $p=0.036$ ) (Table 2). Possible reasons for the significant heterogeneity are different cut-off points and scoring systems of HR estimation because the methods of determining the intensity and quantity of E-cadherin expression varied among individual studies. For example, Ho et al. defined negative E-cadherin immunoexpression as $10 \%$ positive tumor cells or less, and positive E-cadherin immunoexpression was defined more than $10 \%$ positive

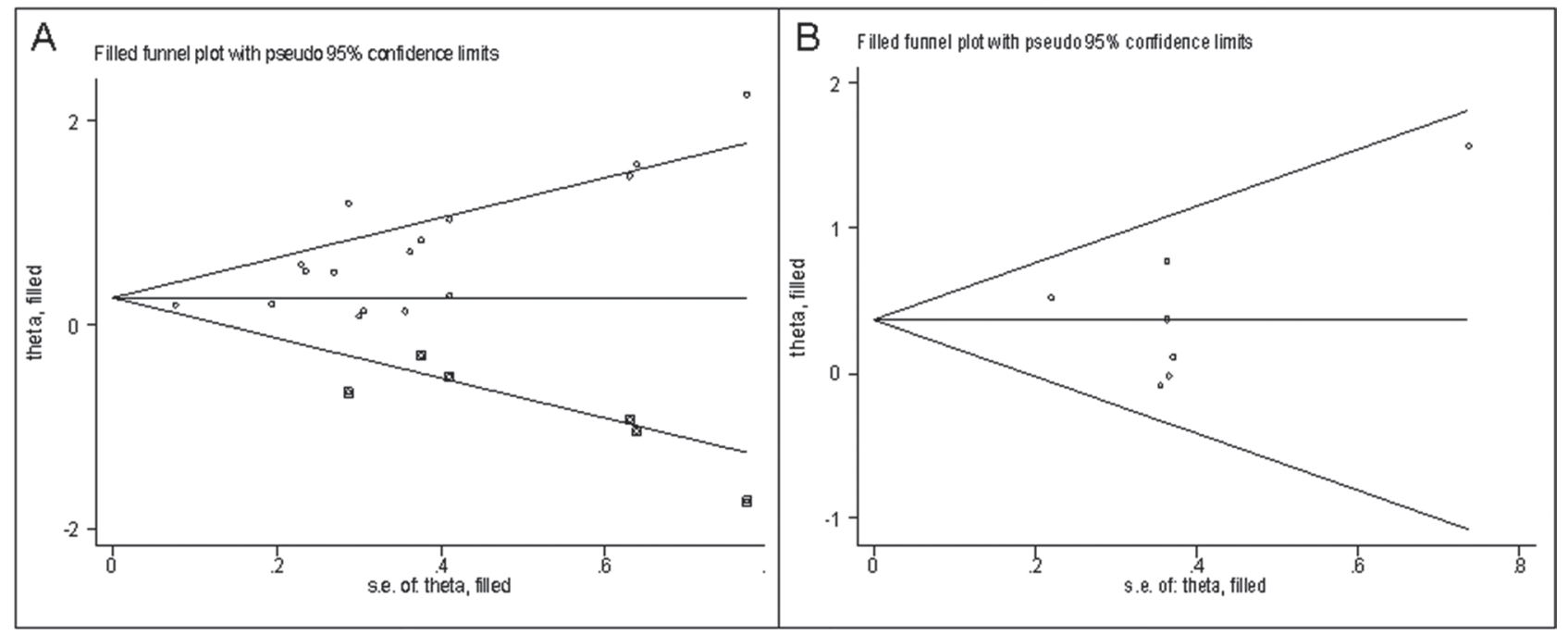

Figure 4: Sensitivity analysis for OS (A) and PFS (B). 
tumor cells [29]. The E-cadherin immunoexpression of the tumors was scored semiquantitatively according to the percentage of positive tumor cells in membranous staining on a 4-point scale of 0 to 4 . However, Taskin et al. considered strong membranous and cytoplasmic staining to be a positive result for E-cadherin. Stained cells were scored with $0 \%$ defined as $0,<1-25 \%$ as $1,25-75 \%$ as 2 , and $>75 \%$ as 3 [28]. The above differences in individual studies might cause variances in HR estimation leading to significant heterogeneity among studies.

Although association between E-cadherin expression and ovarian cancer prognosis has been reported, the contributions of this meta-analysis are as follows: (I) more articles (16 studies vs. 9 studies) and subjects were included in our meta-analysis to provide rigorous evidence compared to a previous meta-analysis conducted in 2012 [22]; (II) stratified analyses of HR estimation, study quality, score criteria, and histological type were conducted to explore heterogeneity; and (III) associations between decreased E-cadherin expression and clinicopathological characteristics were evaluated. Our study reveals that decreased E-cadherin expression has significant associations with FIGO stage, pathologic grade, and residual tumor, which presents a new direction for future research. Moreover, the level of E-cadherin expression was reflective of some clinical characteristics.

Despite the highlights mentioned above, there are still several limitations in our meta-analysis. First of all, the sources of primary antibody and concentration of antibody used in each study varied, which could influence IHC sensitivity. Secondly, the reliability of HR obtained from available data or Kaplan-Meier curves could be affected by inaccuracies in the calculation of censored data. Thirdly, there was no uniform scoring criteria to define the level of E-cadherin expression. Also, cut-off levels for reduced E-cadherin expression varied from 5\% to $25 \%$ without evaluation standards. Finally, significant heterogeneity existed in our study. Although subgroup analyses were conducted, the results do not completely explain the observed heterogeneity.

In conclusion, this study systematically and comprehensively evaluates the prognostic value of decreased E-cadherin expression in ovarian cancer. Poor OS and PFS are significantly related to decreased expression of E-cadherin in ovarian cancer. Decreased E-cadherin expression is correlated with some clinicopathological characteristics including residual tumor size, FIGO, E-cadherin membranous staining, and surgery. Our meta-analysis demonstrates that decreased expression of E-cadherin can be a predictive biomarker of poor prognosis and a critical therapeutic target for ovarian cancer patients.

\section{MATERIALS AND METHODS}

\section{Search strategy}

This meta-analysis was performed in accordance with the Preferred Reporting Items for Systematic Reviews and Meta-Analysis (PRISMA) guidelines [41]. We conducted a search using PubMed, EMBASE and Cochrane Library databases for original studies that evaluated the prognostic value of decreased E-cadherin expression in ovarian cancer. The last search was conducted on March 20, 2017. The search keywords were as follows: "ovarian", "neoplasm", "tumor", "cancer", "E-cadherin", "E-CAD", “CDH1”, “cadherin-1", "prognostic factor", and "survival" . Furthermore, the reference lists of related review articles were screened. This meta-analysis was conducted based on previously published articles. Therefore, ethical approval and patient consent were not required.

\section{Inclusion criteria}

Articles were considered eligible if they met the following criteria: (1) patients diagnosed with ovarian

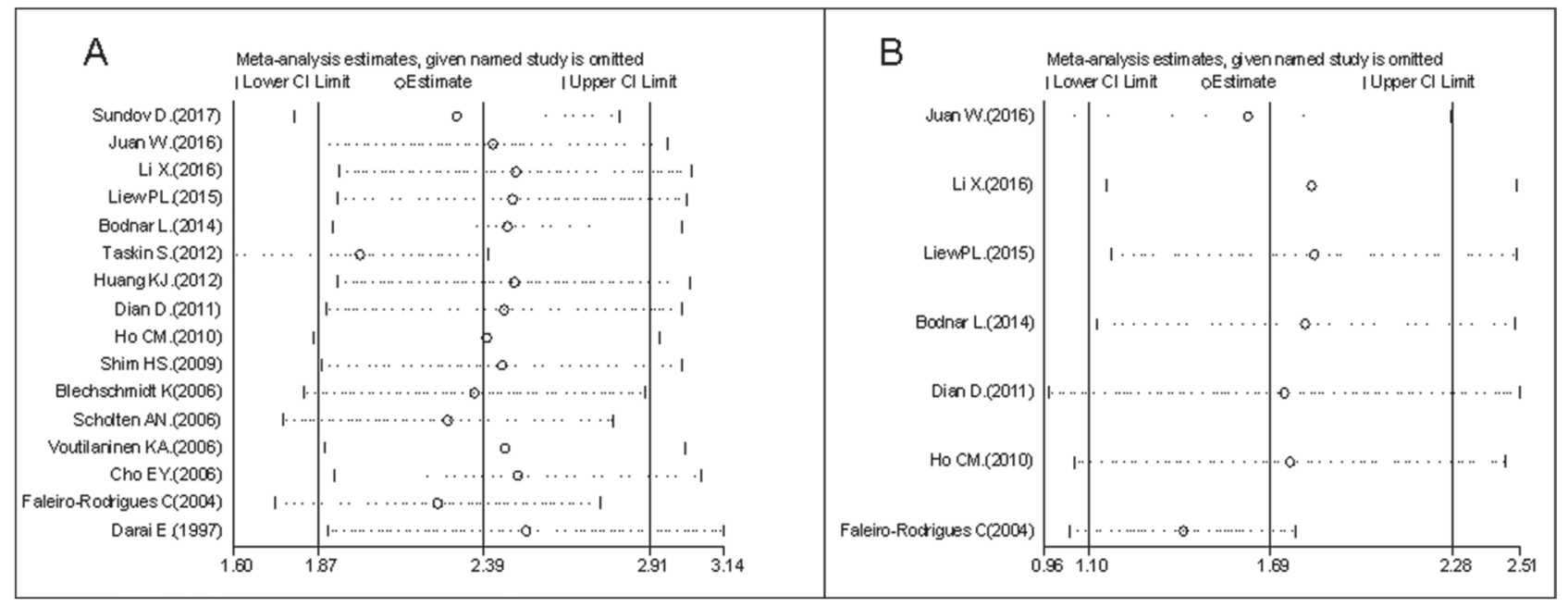

Figure 5: Funnel plot of publication bias for OS (A) and PFS (B). 
cancer using pathological and histological examinations; (2) the level of E-cadherin expression was detected in tumor tissues; (3) original full articles published in English; and (4) articles reported hazard ratio (HR) value and $95 \%$ confidence intervals (CIs) directly or calculated from demographic data or survival curves. Studies with more details and larger sample sizes were only selected if duplicate data from other articles occurred. Reviews, letters, conference abstracts, and comments were excluded.

\section{Quality assessment}

The Newcastle-Ottawa quality assessment scale (NOS) was used to evaluate the quality of included studies by two independent investigators. Three perspectives (selection, comparability, and outcomes) were assessed in accordance with NOS. The quality scores of articles ranged from 0 to 9.0 : scores $\geq 7.0$ indicate high quality.

\section{Data extraction}

According to the inclusion criteria listed above, two independent researchers (Lili Yu and Xiaoli Hua) extracted the following data: the first author, nation, year of publication, mean age of the patients, FIGO stage, case number, mean follow-up period, cut-off level, detecting method, survival type, method for HR estimation, HR with $95 \%$ CIs, histological type, and pathologic type. We resolved any inconsistencies through negotiation and discussion.

\section{Statistical analysis}

Stata Version 12.0 (Stata Corporation, College Station, TX, USA) was used for statistical analysis. HR with $95 \%$ CIs was used to evaluate the prognostic value of decreased E-cadherin expression in ovarian cancer. HR estimation for OS and PFS were directly obtained in some articles. For the studies displaying survival rates with $p$ values from log-rank tests or Kaplan-Meier survival curves, HR could also be extrapolated using the method of Parmar and Tierney [42]. The Chi-square-based $Q$ test and $I^{2}$ were applied as the assessment of heterogeneity across the studies. An $I^{2}<50 \%$ and $p>0.05$ were considered to be homogeneity, in which case the fixed-effect model was used for analysis. If severe heterogeneity was present at $I^{2}>50 \%$ or $p<0.05$, the random-effect model was used. Subgroup analysis and meta-regression were conducted to explore the source of the heterogeneity across the studies [43]. Visual inspection of the funnel plot and Egger's test were done to evaluate the publication bias $(p<0.05$ was statistically significant) $[44,45]$. Sensitivity analysis was carried out to evaluate the robustness of the pooled results by sequential omission of individual studies. If univariate and multivariate analyses were both obtainable, the latter was chosen.

\section{ACKNOWLEDGMENTS AND FUNDING}

This study was supported by the grants from the National Natural Science Foundation of China (No. 81503427 and No. 81372807) and Natural Science Foundation of Hubei Province (No. 2015CKB751).

\section{CONFLICTS OF INTEREST}

All of the authors have declared that no conflicts of interest exist.

\section{REFERENCES}

1. Coleman MP. Cancer survival: global surveillance will stimulate health policy and improve equity. Lancet. 2014; 383:564-573.

2. Coleman MP. Cancer survival in the developing world. Lancet Oncol. 2010; 11:110-111.

3. Rosendahl M, Hogdall CK, Mosgaard BJ. Restaging and Survival Analysis of 4036 Ovarian Cancer Patients According to the 2013 FIGO Classification for Ovarian, Fallopian Tube, and Primary Peritoneal Cancer. Int J Gynecol Cancer. 2016; 26:680-687.

4. Terzi A, Aktas IY, Dolgun A, Ayhan A, Kucukali T, Usubutun A. Early stage epithelial ovarian cancers: a study of morphologic prognostic factors. Pathol Res Pract. 2013; 209:359-364.

5. Feigenberg T, Clarke B, Virtanen C, Plotkin A, Letarte M, Rosen B, Bernardini MQ, Kollara A, Brown TJ, Murphy KJ. Molecular profiling and clinical outcome of high-grade serous ovarian cancer presenting with low- versus highvolume ascites. Biomed Res Int. 2014; 2014:367103.

6. Coticchia CM, Yang J, Moses MA. Ovarian cancer biomarkers: current options and future promise. J Natl Compr Canc Netw. 2008; 6:795-802.

7. Krzystyniak J, Ceppi L, Dizon DS, Birrer MJ. Epithelial ovarian cancer: the molecular genetics of epithelial ovarian cancer. Ann Oncol. 2016; 27:i4-i10.

8. Reddy P, Liu L, Ren C, Lindgren P, Boman K, Shen Y, Lundin E, Ottander U, Rytinki M, Liu K. Formstion of E-cadherin-mediated cell-cell adhesion activates AKT and mitogen activated protein kinase via phosphatidylinositol 3 kinase and ligand-independent activation of epidermalgrowth factor receptor in ovatian cancer cells. Mol Endocrinol. 2005; 19:2564-78.

9. Li Z, Yin S, Zhang L, Liu W, Chen B. Prognostic value of reduced E-cadherin expression in breast cancer: a metaanalysis. Oncotarget. 2017; 8:16445-16455. http://doi. org/10.18632/oncotarget.14860.

10. Frixen UH, Behrens J, Sachs M, Eberle G, Voss B, Warda A, Lochner D, Birchmeier W. E-cadherin-mediated cell-cell adhesion prevents invasiveness of human carcinoma cells. J Cell Biol. 1991; 113:173-185.

11. Umbas R, Schalken JA, Aalders TW, Carter BS, Karthaus HF, Schaafsma HE, Debruyne FM, Isaacs WB. Expression 
of the cellular adhesion molecule E-cadherin is reduced or absent in high-grade prostate cancer. Cancer Res. 1992; 52:5104-5109.

12. Liu X, Chu KM. E-cadherin and gastric cancer: cause, consequence, and applications. Biomed Res Int. 2014; 2014:637308.

13. So WK, Cheng JC, Liu Y, Xu C, Zhao J, Chang VT, Leung PC. Sprouty4 mediates amphiregulin-induced downregulation of E-cadherin and cell invasion in human ovarian cancer cells. Tumour Biol. 2016; 37:9197-9207.

14. Goodwin M, Yap AS. Classical cadherin adhesion molecules: coordinating cell adhesion, signaling and the cytoskeleton. J Mol Histol. 2004; 35:839-844.

15. Wang Q, Wang B, Zhang YM, Wang W. The association between CDH1 promoter methylation and patients with ovarian cancer: a systematic meta-analysis. J Ovarian Res. 2016; 9:23.

16. Sakuragi N, Nishiya M, Ikeda K, Ohkouch T, Furth EE, Hareyama H, Satoh C, Fujimoto S. Decreased E-cadherin expression in endometrial carcinoma is associated with tumor dedifferentiation and deep myometrial invasion. Gynecol Oncol. 1994; 53:183-189.

17. Nomura E, Sakuragi N, Fujimoto S. [Abnormal E-cadherin expression as a risk factor for deep myometrial invasion and lymph node metastasis in endometrial carcinoma]. [Article in Japanese]. Nihon Rinsho. 1995; 53:1607-1612.

18. Huang KJ, Sui LH. The relevance and role of vascular endothelial growth factor C, matrix metalloproteinase-2 and E-cadherin in epithelial ovarian cancer. Med Oncol. 2012; 29:318-323.

19. Dian D, Bruning A, Mylonas I. E-cadherin as a prognostic marker in human serous carcinomas of the ovary: an immunohistochemical analysis. Arch Gynecol Obstet. 2011; 284:437-443.

20. Voutilainen KA, Anttila MA, Sillanpaa SM, Ropponen KM, Saarikoski SV, Juhola MT, Kosma VM. Prognostic significance of E-cadherin-catenin complex in epithelial ovarian cancer. J Clin Pathol. 2006; 59:460-467.

21. Wang Q, Wang B, Zhang YM, Wang W. The association between $\mathrm{CDH} 1$ promoter methylation and patients with ovarian cancer: a systematic meta-analysis. J Ovarian Res. 2016; 9:23.

22. Peng HL, He L, Zhao X. Association of reduced immunohistochemical expression of E-cadherin with a poor ovarian cancer prognosis--results of a meta-analysis. Asian Pac J Cancer Prev. 2012; 13:2003-2007.

23. Sundov D, Petric MB, Mrklic I, Bacic B, Vrdoljak E, Tomis S. Prognostic significance of MAPK, Topo IIalpha and E-cadherin immunoexpression in ovarian serous carcinomas. Neoplasma. 2017; 64:289-298.

24. Juan W, Shan K, Na W, Rong-Miao Z, Yan L. The Associations of Genetic Variants in E-cadherin Gene With Clinical Outcome of Epithelial Ovarian Cancer. Int J Gynecol Cancer. 2016; 26:1601-1607.
25. Li X, Yang J, Wang X, Li X, Liang J, Xing H. Role of TWIST2, E-cadherin and Vimentin in epithelial ovarian carcinogenesis and prognosis and their interaction in cancer progression. Eur J Gynaecol Oncol. 2016; 1:100-108.

26. Liew PL, Hsu CS, Liu WM, Lee YC, Lee YC, Chen CL. Prognostic and predictive values of Nrf2, Keap1, p16 and E-cadherin expression in ovarian epithelial carcinoma. Int J Clin Exp Pathol. 2015; 8:5642-5649.

27. Bodnar L, Stanczak A, Cierniak S, Smoter M, Cichowicz M, Kozlowski W, Szczylik C, Wieczorek M, LamparskaPrzybysz M. Wnt/beta-catenin pathway as a potential prognostic and predictive marker in patients with advanced ovarian cancer. J Ovarian Res. 2014; 7:16.

28. Taskin S, Dunder I, Erol E, Taskin EA, Kiremitci S, Oztuna D, Sertcelik A. Roles of E-cadherin and cyclooxygenase enzymes in predicting different survival patterns of optimally cytoreduced serous ovarian cancer patients. Asian Pac J Cancer Prev. 2012; 13:5715-5719.

29. Ho CM, Cheng WF, Lin MC, Chen TC, Huang SH, Liu FS, Chien CC, Yu MH, Wang TY, Hsieh CY. Prognostic and predictive values of E-cadherin for patients of ovarian clear cell adenocarcinoma. Int J Gynecol Cancer. 2010; 20:14901497.

30. Shim HS, Yoon BS, Cho NH. Prognostic significance of paired epithelial cell adhesion molecule and E-cadherin in ovarian serous carcinoma. Hum Pathol. 2009; 40:693-698.

31. Blechschmidt K, Sassen S, Schmalfeldt B, Schuster T, Hofler H, Becker KF. The E-cadherin repressor Snail is associated with lower overall survival of ovarian cancer patients. Br J Cancer. 2008; 98:489-495.

32. Scholten AN, Aliredjo R, Creutzberg CL, Smit VT. Combined E-cadherin, alpha-catenin, and beta-catenin expression is a favorable prognostic factor in endometrial carcinoma. Int J Gynecol Cancer. 2006; 16:1379-1385.

33. Cho EY, Choi Y, Chae SW, Sohn JH, Ahn GH. Immunohistochemical study of the expression of adhesion molecules in ovarian serous neoplasms. Pathol Int. 2006; 56:62-70.

34. Faleiro-Rodrigues C, Macedo-Pinto I, Pereira D, Lopes CS. Prognostic value of E-cadherin immunoexpression in patients with primary ovarian carcinomas. Ann Oncol. 2004; 15:1535-1542.

35. Darai E, Scoazec JY, Walker-Combrouze F, Mlika-Cabanne N, Feldmann G, Madelenat P, Potet F. Expression of cadherins in benign, borderline, and malignant ovarian epithelial tumors: a clinicopathologic study of 60 cases. Hum Pathol. 1997; 28:922-928.

36. Gumbiner BM. Cell adhesion: the molecular basis of tissue architecture and morphogenesis. Cell. 1996; 84:345-357.

37. Zhang Y, Zhao Y, Jiang G, Zhang X, Zhao H, Wu J, Xu $\mathrm{K}$, Wang E. Impact of p120-catenin isoforms $1 \mathrm{~A}$ and $3 \mathrm{~A}$ on epithelial mesenchymal transition of lung cancer cells expressing E-cadherin in different subcellular locations. Plos One. 2014; 9:e88064. 
38. Ashaie MA, Chowdhury EH. Cadherins: The Superfamily Critically Involved in Breast Cancer. Curr Pharm Des. 2016; 22:616-638.

39. Nantajit D, Lin D, Li JJ. The network of epithelialmesenchymal transition: potential new targets for tumor resistance. J Cancer Res Clin Oncol. 2015; 141:1697-1713.

40. Kong D, Li Y, Wang Z, Sarkar FH. Cancer Stem Cells and Epithelial-to-Mesenchymal Transition (EMT)-Phenotypic Cells: Are They Cousins or Twins? Cancers (Basel). 2011; 3:716-729.

41. Moher D, Liberati A, Tetzlaff J, Altman DG. Preferred reporting items for systematic reviews and meta-analyses: the PRISMA statement. Plos Med. 2009; 6:e1000097

42. Parmar MK, Torri V, Stewart L. Extracting summary statistics to perform meta-analyses of the published literature for survival endpoints. Stat Med. 1998; 17:2815-2834.

43. Hardy RJ, Thompson SG. Detecting and describing heterogeneity in meta-analysis. Stat Med. 1998; 17:841-856.

44. Egger M, Davey SG, Schneider M, Minder C. Bias in metaanalysis detected by a simple, graphical test. BMJ. 1997; 315:629-634.

45. Sterne JA, Gavaghan D, Egger M. Publication and related bias in meta-analysis: power of statistical tests and prevalence in the literature. J Clin Epidemiol. 2000; 53:1119-1129. 